\title{
Diseño para servir
}

\author{
Vishwa N.P. Mathur ${ }^{1}$ \\ Eliseo Temprano ${ }^{2}$
}

\section{RESUMEN}

Las estructuras de madera se han usado en América del Norte desde tiempos remotos. Después de la llegada de los primeros inmigrantes europeos se desarrolló en esta parte del continente el sistema de construcción a base de bastidores ligeros. El tema central del artículo es exponer una serie de ideas acerca de las mejores formas de aprovechar las características y procesos de la madera para lograr diseños exitosos. Las tres reglas principales para obtener el mejor comportamiento de la madera en estructuras son: 1) Usar madera seca; 2) mantenerla seca y 3) tratarla con un preservador adecuado cuando la madera pueda estar expuesta al ataque de organismos. El artículo está ilustrado con varios ejemplos arquitectónicos de estructuras de madera en Canadá los cuáles dan una idea de la gran variedad de soluciones disponibles para el arquitecto, la flexibilidad del sistema y su adaptabilidad a diversas situaciones y contextos.

\section{PALABRAS CLAVES}

Estructuras ligeras de madera, madera en servicio, protección de las estructuras de madera, tratamientos de preservación.

\begin{abstract}
Wood structures have been used in North America since prehistoric times. Only after the arrival of the first European settlers, the light frame structure system was developed in this part of the continent. This paper presents a series of ideas about the best ways to make more adequate use of wood characteristics and about the necessary processes to obtain successful designs. The three main rules for achieving maximum performance in the use of wood in structures are: 1) Use dry wood, 2) keep it dry and 3) treat the wood with a suitable preservative to protect it from destructive organisms. Architectural examples of wood buildings in Canada are provided and these give an idea of the great variety of solutions available to architects, the flexibility of the proposed systems and their adaptability with respect to a wide variety of situations and types of construction.
\end{abstract}

\section{KEY WORDS}

Light-frame structures, wood in service, timber structure protection, preservative treatments.

1 Consejero Científico. Servicio Forestal. Departamento de Recursos Naturales de Canadá. Place Vincent Massey $\bullet 351$ St. Joseph Boulevard $\bullet 20^{\text {th }}$ Floor $\bullet$ Hull, Quebec $\bullet$ K1A 1 G5

2 Director de Temprano Associates Inc. Architects \& Planners. 201 Rideau Street $\bullet$ 2nd Floor Ottawa, Ontario, Canadá $\bullet$ K1N 5X8.

Recibido para su publicación el 16 de Marzo de 1995 
Por milenios, la madera ha sido utilizada como material de construcción por los nativos de Norte y Sudamérica. La llegada de los europeos en el siglo XIV, dio como resultado que se adoptaran los sistemas a base de mampostería que tuvieron sus orígenes en la época de los romanos. Muchas culturas indígenas de América han continuado con el uso de la madera como un material de construcción primario. Normalmente han sido viviendas y algunas estructuras ocasionales para fines públicos o comunales. La tecnología norteamericana para estructuras ligeras es el resultado de la fusión de la tradición indígena en construcción, con el enfoque de los sistemas modernos. De muchas formas, esto representa la evolución de una tradición en construcción vigente, adaptada a la realidad de la sociedad y vida moderna.

Una de las principales razones para el uso continuo y exitoso de la madera como material de construcción, es su gran flexibilidad y adaptabilidad, que responde a una variedad de condiciones y estilos. La clave para triunfar en la construcción con madera reside en entender sus características particulares. Sólo con el conocimiento adecuado y la aplicación de esta tecnología se puede garantizar el éxito en este campo. Cuando se da un cruce de culturas en la aplicación de tecnología, es sumamente importante que este conocimiento sea transferido, no trasplantado. Dicha transferencia debe darse a través del filtro del entendimiento de las condiciones locales o regionales. La Comisión Forestal para América del Norte (COFAN) en su espíritu de cooperación, ha patrocinado el desarrollo de un Manual que combina lo mejor de las experiencias de los Estados Unidos, Canadá y México para esta tecnología para garantizar su correcta aplicación en México. La preparación de este Manual sobre Estructuras Ligeras, es sólo el inicio de esta etapa. La tarea más importante de transferir tecnología a los usuarios inicia con la realización de seminarios y distribución del Manual entre los profesionales de la construcción. La retroalimentación que se obtenga de exponerlo a ingenieros, arquitectos y especialistas de la industria de la construcción, contribuirá a mantenerlo actualizado y relevante para futuras ediciones.

A continuación se describen algunas de las recomendaciones más importantes de cómo la madera debe manejarse para lograr el máximo rendimiento. Esto les permitirá comprender la naturaleza del material.

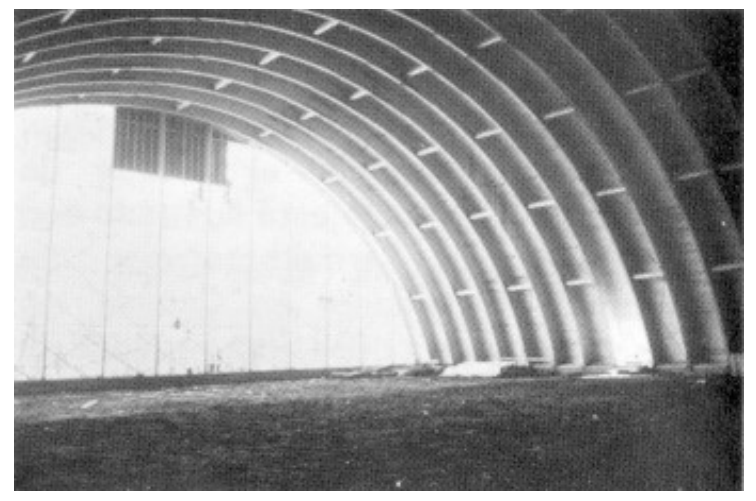

Estructura laminada para grandes claros

Los ingenieros y los arquitectos tienen que diseñar con el objetivo de servir al usuario de la manera más eficiente y efectiva. Hay oportunidades arquitectónicas-oportunidades de lograr el máximo rendimiento de la madera bajo condiciones difíciles. Tomando como base la experiencia internacional, actual y pasada, el servicio de las estructuras de madera se puede prolongar mediante buenos diseños y buena prácticas. Hay 
dos reglas básicas para obtener el mejor servicio de la madera bajo cualquier condición: usar madera seca $y$ mantenerla seca.

Las reglas son simples, pero no siempre se pueden cumplir. Por ello se debe considerar una tercera regla, aplicable sólo cuando la condición se usar madera seca no se pueda cumplir: tratarla con un preservador adecuado.

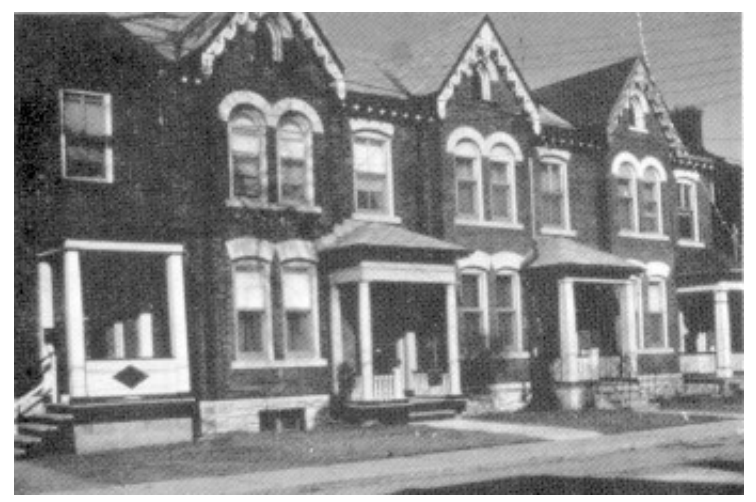

Casa habitación con estructura de madera de casi 100 años de antiguedad recubierta con materiales pétreos

\section{Usar madera seca}

La presencia de humedad en la madera es realmente la clave para la durabilidad de las estructuras. Sin una fuente de humedad, aún la madera más susceptible a la pudrición, puede ser garantizada para uso a largo plazo; pero si la madera se utiliza con humedad por arriba del punto de saturación de la fibra (contenido de humedad $=30 \%$ ), ésta fallará con el transcurso del tiempo. Los arquitectos y los ingenieros deben tener bien claro que a menos que se especifique madera seca en estufa, es muy posible que la madera se surta en condición verde, con contenido de humedad por arriba del $30 \%$. Debe tenerse en cuenta que el uso de madera sin secar, no sólo presenta problemas de pudrición sino también de cambio de dimensiones y forma (contracciones y distorsiones) en las estructuras y viguetas, ya que de hecho el material se seca estando ya en servicio.

\section{Mantenerla seca}

La madera debe mantenerse seca desde que sale de la estufa, hasta que se termina la estructura, transportándola y almacenándola bajo cubierta y separada del suelo. Comúnmente, se utilizan embalajes para vigas laminadas y madera seca, aunque también se pueden emplear otro tipo de envolturas. Es primordial evitar que la madera seca sea manejada sin cuidado en lugares húmedos o fangosos.

Cuando la madera en servicio está expuesta en exteriores, el problema de mantenerla razonablemente seca varía con el clima local y el uso de los elementos de madera. La temperatura y la precipitación pluvial son factores importantes. La orientación y la sombra de otras construcciones o árboles puede contribuir a que la madera permanezca húmeda por períodos prolongados. Los puntos críticos de absorción de humedad son: los cantos de los granos expuestos; los huecos de los capilares entre las superficies; conexiones; bolsas, grietas y hendiduras de las superficies longitudinales. Las generaciones anteriores de diseñadores han proporcionado un valioso legado de detalles sobre el uso de la madera. Pero las lecciones que aprendieron estas generaciones, con frecuencia se escapan por la rapidez con que se construye actualmente, permitiendo que, nuevamente se resuelvan los mismos problemas. 
A continuación se dan algunos lineamientos que han sido útiles para conservar por mucho tiempo estructuras totalmente expuestas:

a) La inclinación de las secciones transversales en dirección opuesta a los rayos solares y a la lluvia. Proteger los extremos de postes y otros elementos verticales.

b) Facilitar la circulación del aire y un drenaje natural. Cuando se diseñe, largueros $u$ otros detalles, deben estar protegidos de la lluvia y permitir un secado rápido después de mojados, de esta manera se disminuirán considerablemente las condiciones favorables para el desarrollo de la pudrición. Todos los barandales, escaleras y cubiertas de terrazas, viguetas o vigas deberán tener una cierta pendiente. Evite los huecos en los capilares que puedan retener agua. Deje una separación en las juntas a tope en barandales y terrazas para permitir la circulación del aire.

c) Utilice especies y calidades de madera que sean estables dimensionalmente estando en servicio. Madera limpia con límite en la inclinación de la fibra, si se consigue, es mucho más recomendable (aunque más cara). Escoja piezas de madera con secciones que sean lo más cuadradas posible, en lugar de piezas anchas y delgadas; por ejemplo, de $3 \times 4$ pulg en vez de 2 × 6 ó 1 x 8 pulg o utilizar madera aserrada en dirección radial, si esto es posible.

d) Asegure bien las estructuras, utilizando conectores galvanizados 0 son un recubrimiento anti-corrosivo o adhesivos a prueba de agua.

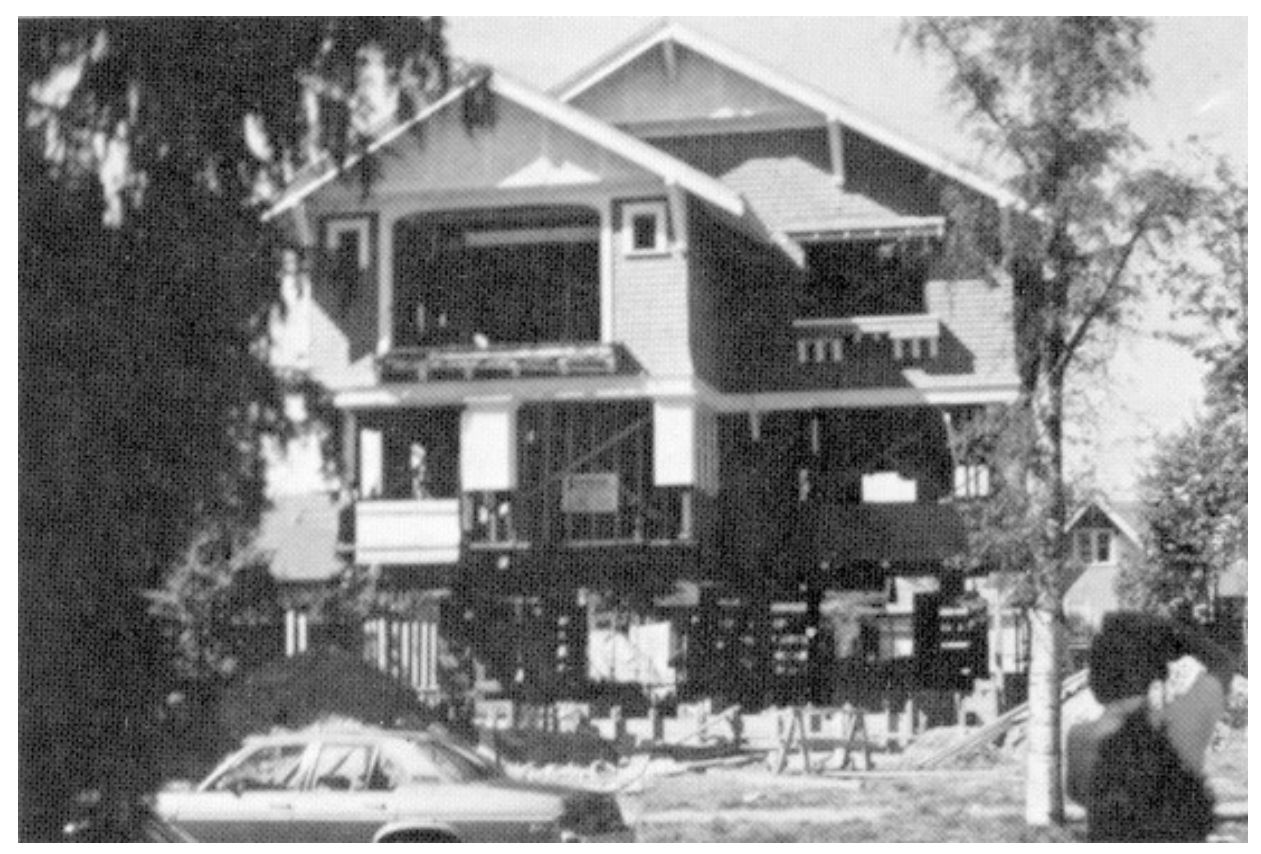

Casa con nivel nuevo integrado en la planta baja (la estructura original se elevó temporalmente para construir el nivel inferior) 
e) Utilice duramen de especies resistentes a la pudrición para una máxima durabilidad a la intemperie. Por ejemplo la madera de "Western red cedar", abundante en Canadá, se usa comúnmente por su durabilidad y estabilidad; el duramen de cualquier especie, es por regla general, más resiente a al pudrición que la albura, aunque menos que la especie arriba citada.

f) El uso de técnicas de acabados bien diseñados y con buen mantenimiento, con la aplicación previa de un preservador repelente al agua por inmersión, cuidando que los extremos, recortes y todas las superficies expuestas sean cubiertos, ayudan a prolongar la vida útil de la madera en exteriores al disminuir la tasa de humedecimiento. Existe hoy día, la tendencia hacía el uso de tintas que penetran en la madera en vez de pinturas ya que a las primeras se les puede dar mantenimiento más fácilmente.

En resumen, el arquitecto tiene la oportunidad de mejorar el comportamiento de los elementos de madera en exteriores, minimizando la exposición a la humedad y sus efectos.

\section{Tratarla con un preservador adecuado}

La primera y mejor manera de protección radica en un diseño arquitectónico correcto que prevenga el humedecimiento de la madera en servicio. Si esto es difícil o imposible de tener, se requiere alguna forma de protección química mediante el uso de un preservador adecuado que dé la máxima vida en servicio.

El servicio debe considerarse de acuerdo a la necesidad de preservación y siempre que se evalúe el riesgo al que puede estar sujeta la madera sin tratar.
Esta evaluación se basa en la incidencia de riesgo 0 daño y sus posibles consecuencias, en términos de daño, costo de reparación y los inaceptables trastornos que ocasionan durante la reparación. El peligro entonces debe considerarse como el resultado de las consecuencias del número de riesgos. Se pueden definir cuatro categorías sobre la necesidad de un tratamiento preservador:

a. Necesidad fundamental de preservación. En donde la madera esté expuesta a un ambiente continuo de riesgo $y$ no se pueda proteger mediante diseño, en donde las normas de construcción indiquen tratamiento, 0 en donde la experiencia muestre que hay un alto riesgo de falla en estructuras que soportan carga y que puede representar un daño serio a la vida o propiedad.

b. Preservación deseable. En donde la experiencia haya mostrado que existe un alto riesgo de falla por errores en el diseño o en la mano de obra o en donde haya riesgo de ataque, el cual si ocurre sería difícil y costoso de remediar.

c. Preservación opcional. En donde existe un bajo riesgo al biodeterioro (ataque por hongos y/o insectos), o en donde la acción correctiva 0 reemplazo es sencilla. En esta situación, el costo de preservar antes puede sopesarse comparando contra la posibilidad de que reparaciones posteriores sean más costosas.

d. Preservación innecesaria. En donde las condiciones de servicio hagan poco probable que la madera esté en riesgo. 


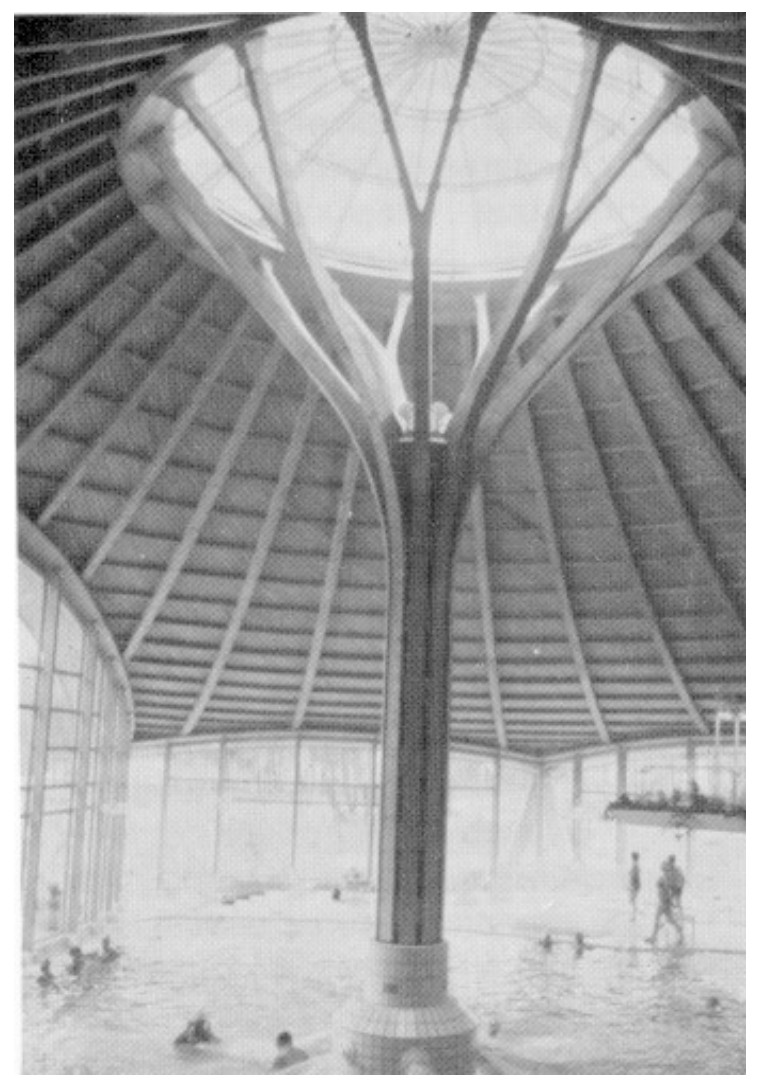

Cubierta de madera laminada para alberca
El Manual COFAN presenta varias opciones de preservadores que están disponibles y aprobados para su uso en México. En donde la exposición a la intemperie y el contacto con el suelo no se pueda evitar, la madera tratada con preservadores debe especificarse para una máxima vida en servicio. El duramen sin tratar de especies durables, puede utilizarse en estas situaciones, aunque esto llevaría a una menor vida útil de la estructura.

Mediante el uso de las ayudas de diseño, recomendaciones y especificaciones del Manual COFAN, es posible esperar que la construcción de viviendas con madera en México, sea bella y durable, cuando arquitectos $e$ ingenieros diseñen y construyan apropiadamente. Las soluciones que emanen de las aplicaciones específicas son muy variables y están limitadas sólo por la imaginación de los arquitectos. 